\title{
Ki-67 and breast cancer prognosis: does it matter if Ki-67 level is examined using preoperative biopsy or postoperative specimen?
}

\author{
Soon Bo Choi ${ }^{1}$ (D) Jung Min Park ${ }^{1}$. Jee Hyun Ahn ${ }^{1}$. Jieon Go ${ }^{1}$. Jeeye Kim ${ }^{1}$. Hyung Seok Park ${ }^{1}$. Seung II Kim ${ }^{1}$. \\ Byeong-Woo Park ${ }^{1} \cdot$ Seho Park ${ }^{1}$ (i)
}

Received: 14 November 2021 / Accepted: 4 January 2022 / Published online: 13 January 2022

(c) The Author(s) 2022

\begin{abstract}
Purpose This study aimed to identify the association between Ki-67 level and the prognosis of patients with breast cancer, regardless of the timing of Ki-67 testing (using preoperative biopsy vs. postoperative specimen).

Methods A total of 4177 patients underwent surgery between January 2008 and December 2016. Immunohistochemical Ki-67 levels, using either preoperative (1673) or postoperative (2831) specimens, were divided into four groups using cutoff points of $10 \%, 15 \%$, and $20 \%$.

Results Groups with higher-Ki-67 levels, in both the pre- and postoperative periods, showed significantly larger tumor size, higher grade, more frequent hormone receptor-negativity and human epidermal growth factor receptor 2 overexpression, and active adjuvant treatments than groups with lower-Ki-67 levels. High-Ki-67 levels were also significantly associated with poor survival, irrespective of the timing of specimen examination.

Conclusion Despite the problems associated with Ki-67, Ki-67 level is an important independent prognostic factor, regardless of the timing of Ki-67 testing, i.e., preoperative or postoperative testing.
\end{abstract}

Keywords Ki-67 $\cdot$ Breast cancer $\cdot$ Prognosis $\cdot$ Biopsy $\cdot$ Surgical specimen

$\begin{array}{ll}\text { Abbreviations } \\ \text { BMI } & \text { Body mass index } \\ \text { CNB } & \text { Core needle biopsy } \\ \text { DFS } & \text { Disease-free survival } \\ \text { ER } & \text { Estrogen receptor-positive } \\ \text { FISH } & \text { Fluorescence in situ hybridization } \\ \text { IHC } & \text { Immunohistochemical } \\ \text { OS } & \text { Overall survival } \\ \text { PR } & \text { Progesterone receptor } \\ \text { SD } & \text { Standard deviation } \\ \text { SISH } & \text { Silver in situ hybridization } \\ \text { VAB } & \text { Vacuum-assisted biopsy }\end{array}$

Seho Park

PSH1025@yuhs.ac

Soon Bo Choi

csbnhi@yuhs.ac

1 Division of Breast Surgery, Department of Surgery, Yonsei University College of Medicine, Seoul, Republic of Korea

\section{Introduction}

Breast cancer is a heterogeneous disease [1-3]. The nature of breast cancer varies not only between different histologic types or subtypes but also varies from person-to-person. Presently, the treatment of breast cancer requires a multifaceted approach and early detection of breast cancer is crucial $[4,5]$. Gene profiling and immunohistochemical (IHC) studies are conducted to investigate various biologic characteristics and predict the prognoses of breast cancer patients [4]. At present, four breast cancer subtypes have been identified, namely, luminal A, luminal B, human epidermal growth factor receptor 2 (HER2) enriched, and basal-like. Accordingly, various treatment strategies have been implemented and the prognosis has been predicted [4-6].

Among various characteristics of breast cancer, progression and aggression are currently evaluated using the biologic indicator Ki-67 to determine the degree of tumor proliferation [7]. Ki-67 is a non-histone nuclear cortex protein, which is encoded by the MKI67 gene and is expressed in the cell nucleus during the $\mathrm{G} 1, \mathrm{~S}, \mathrm{G} 2$, and $\mathrm{M}$ phases of the cell cycle, but not in the G0 phase $[8,9]$. 
Since 2007, many studies have been conducted to determine the cutoff value of Ki-67 that can be used as a prognostic factor [10-13]. Based on these studies, in the 2011 St. Gallen conference, Ki-67 level became the standard factor for differentiating between luminal B and luminal A types in patients with estrogen receptor-positive $(E R+)$ and HER2-negative (HER2-) breast cancer; the recommended cutoff value for this classification was 14\% [6]. However, the accuracy, analytical validity, and reproducibility of the methods for detecting Ki-67 remained controversial [9]. Therefore, for determining its analytical validity, the international Ki-67 in the Breast Cancer Working Group has provided guidelines for Ki-67 scoring [14]. Nevertheless, challenges with global standardization still persist and the 2015 St. Gallen conference recommended that a lab-specific median Ki-67 cutoff value should be determined through standardization procedures in each institution [15].

Keeping the analytical problems aside, the timing of the test also remains questionable, making its reproducibility debatable [9]. Although there have been previous studies on the concordance of preoperative and postoperative $\mathrm{Ki}-67$ levels of only the same patient, it remains questionable whether the analysis of Ki-67 level performed at one time can represent the entire proliferation index of breast cancer [16-19]. In addition, several studies have tackled the timing of only the test pathologically; however, information on clinical approaches remains scarce [17-19].

The purpose of this study was to resolve the question on the suitable timing of Ki-67 testing and to evaluate whether the Ki-67 index is an appropriate proliferation index of breast cancer, i.e., a factor influencing the prognosis of breast cancer, regardless of the time of testing.

\section{Methods}

\section{Patients}

We retrospectively reviewed the records of 4177 patients with pathologically confirmed invasive breast cancer who underwent surgery at the Department of Surgery, Yonsei University College of Medicine, between January 2008 and December 2016. Patients whose Ki-67 levels were investigated for breast carcinoma were included, regardless of when the Ki-67 levels were examined. We excluded patients for whom Ki-67 was not assessed, those who underwent neoadjuvant chemotherapy, and patients with in situ carcinoma, microinvasive carcinoma, recurrent, or metastatic disease. The study population comprised patients with stage I-IIIc breast cancer according to the American Joint Committee on Cancer, 8th edition, which is a commonly used pathological staging system.

The time of measuring Ki-67 levels was divided into preand postoperative periods. Preoperative Ki-67 (Pre-Ki-67) was defined as Ki-67 levels measured in tissues obtained through core needle biopsy (CNB), vacuum-assisted biopsy (VAB), or rarely excisional/incisional biopsy. Further, postoperative Ki-67 (Post-Ki-67) was defined as Ki-67 levels measured in tissue specimens obtained from surgery. IHC analysis was performed on biopsy and permanent specimen. Ki-67 levels were arbitrarily stratified and patients were divided into four subgroups according to $\mathrm{Ki}-67$ level: $<10 \%$ (lowest), 10- <15\% (medium-low), 15-<20\% (medium-high), and $\geq 20 \%$ (highest).

This study was approved by the Institutional Review Board of Severance Hospital, Seoul, Republic of Korea (IRB No. 4-2020-1293). The requirement for informed consent was waived due to the retrospective nature of the study.

\section{Clinicopathological characteristics}

Patient demographics and clinical information including treatment modalities and expression of ER, progesterone receptor (PR), HER2, and Ki-67 levels were obtained from electronic medical records and pathology reports. Positive ER and PR were defined as IHC nuclear-stained cells $\geq 1 \%$, based on the American Society of Clinical Oncology/College of American Pathologists (ASCO/CAP) guidelines [20]. According to the ASCO/CAP guidelines, HER2 was scored as $0,1+, 2+$, or $3+$ [21]. In patients with an HER2 $2+$ result, fluorescence in situ hybridization (FISH) or silver in situ hybridization (SISH) was performed. HER2 gene amplification was defined as a HER2 gene/chromosome 17 copy number ratio $\geq 2.0$ or a HER 2 gene/chromosome 17 copy number ratio $<2.0$, but with average HER 2 copy number $\geq 6.0$ signals/cell according to the ASCO/CAP guidelines [21]. HER2 was considered positive in cases with an IHC score of $3+$ or gene amplification detected by FISH or SISH.

IHC was performed for measuring Ki-67 levels in both preoperative biopsy tissue and postsurgical specimens using a primary MIB-1 antibody (Dako Denmark A/S, Glostrup, Denmark), following protocols established by the Department of Pathology at our institution. Using a visual grading system, a specialized pathologist highly experienced in breast pathology determined the Ki-67 levels by counting the number of positively stained nuclei on hotspot and expressed it as a percentage of total tumor cells in the specimen (Ki-67 level; \%).

\section{Endpoints}

The primary endpoint was disease-free survival (DFS) and overall survival (OS) in relation to the four subgroups of Pre-Ki-67 and Post-Ki-67 levels. Recurrence was divided into loco-regional recurrence and distant metastasis. Contralateral breast cancer was not included as a recurrence. The follow-up period was set from the day of surgery to the date 
of the last hospital visit, regardless of the department visited. It was possible to confirm the exact patient's event by following up regardless of the department. DFS was defined as the period from the date of surgery to the date of the first observation of recurrence, death, or the last follow-up date without evidence of any events, while OS was defined as the period from the date of surgery to the date of death or the last follow-up.

\section{Statistical analyses}

The clinicopathological information, including demographic, clinical, and treatment-related data of the patients, were analyzed based on the four subgroups for Pre-Ki-67 and Post-Ki-67 levels. The variables were compared using a Chi-squared test, $t$ test, or analysis of variance. The Kaplan-Meier method was used to predict survival rates, and a log-rank test was used to compare the four subgroups. In a multivariate analysis, Cox proportional hazard regression was used for adjustment of other factors. Cox regression analysis was used to calculate hazard ratios and $95 \%$ confidence intervals. Values were two-sided and statistical significance was defined at $P<0.05$. All statistical analyses were performed using SPSS software, version 25 (IBM Corp., Armonk, NY, USA).

\section{Results}

\section{Characteristics of patients}

The clinicopathological characteristics of patients are summarized in Table 1. There were 1346 (32.2\%) patients who underwent $\mathrm{Ki}-67$ testing in the preoperative period alone, 2504 (59.9\%) patients who underwent Ki-67 testing in the postoperative period alone, and 327 (7.9\%) patients who underwent Ki-67 testing both in the preoperative and postoperative periods. Finally, a total of 1673 and 2831 patients were included in the Pre-Ki-67 and Post-Ki-67 groups, respectively. The median Ki-67 of the Pre-Ki-67 group was 10.00 and that of the Post-Ki-67 group was 12.55 . The mean \pm standard deviation (SD) of the age of the patients at diagnosis was $52.3 \pm 11.1$ years and the mean \pm SD of body mass index (BMI) was $23.4 \pm 3.4 \mathrm{~kg} / \mathrm{m}^{2}$.

In the Pre-Ki-67 group, the lowest, medium-low, medium-high, and highest Ki-67 levels were found in 770 (46.0\%), 256 (15.3\%), 118 (7.1\%), and 529 patients (31.6\%), respectively, and in the Post-Ki-67 group, the lowest, medium-low, medium-high, and highest Ki-67 levels were found in 995 (35.1\%), 467 (16.5\%), 198 (7.0\%), and 1171 patients $(41.4 \%)$, respectively. The means \pm SDs of age in the Pre-Ki-67 and Post-Ki-67 groups were $52.2 \pm 10.6$ years and $52.6 \pm 11.3$ years, respectively, while the means \pm SDs of BMI in each of the two groups were $23.3 \pm 3.4 \mathrm{~kg} / \mathrm{m}^{2}$ and $23.4 \pm 3.4 \mathrm{~kg} / \mathrm{m}^{2}$, respectively. Age and BMI did not significantly differ with Ki-67 levels in both the Pre-Ki-67 and Post-Ki-67 groups.

Regarding pathological parameters, most of the factors showed statistically significant differences according to the Ki-67 levels and similar trends were observed in both groups. Tumors sized $\leq 2 \mathrm{~cm}$, histologic grade I/II, hormone receptor-positivity, and HER2-negativity were significantly associated with lower Ki-67 levels. In contrast, tumors sized $>2 \mathrm{~cm}$, histologic grade III, hormone receptor-negativity, and overexpression of HER2 were significantly associated with the highest Ki-67 levels. Unlike other pathological factors, lymph node status was not significantly related to Ki-67 levels in any period.

Regarding treatment modalities, both periods showed similar trends. The frequency of local therapy did not significantly differ according to Ki-67 levels. However, the use of systemic therapies such as chemo-endocrine therapy and targeted therapy showed significant differences across Ki-67 levels as expected. More than $90 \%$ of the patients, who received endocrine therapy, tended to have the lowest level of Ki-67. In contrast, most patients who received chemotherapy and biologic therapy had medium-high or highest level of Ki-67.

\section{Survival according to Pre-Ki-67 and Post-Ki-67 levels}

Figure 1 shows the 10-year DFS and OS according to Ki-67 levels in both the Pre-Ki-67 and Post-Ki-67 groups. The mean follow-up duration of the patients was 69.3 months (SD: 30.4 months). The mean follow-up durations in the Pre-Ki-67 and Post-Ki-67 groups were 70.7 months (SD: 23.1 months) and 69.5 months (SD: 33.5 months), respectively. DFS and OS were significantly different between the groups and similar trends were observed in each period. Although the remaining three Pre-Ki-67 groups had a worse prognosis compared with the lowest $\mathrm{Ki}-67$ group, there was no statistical significance observed between the lowest and medium-low groups (Fig. 1a and c). Likewise, with regard to DFS and OS of the Post-Ki-67 group, it was confirmed that the three remaining groups all had a worse prognosis compared with that of the lowest group (Fig. 1b and d). However, there was no significant difference between the lowest and low-medium groups in the OS of the Post-Ki-67 group (Fig. 1d).

\section{Multivariate and subgroup analyses}

The results of multivariate analyses for DFS and OS are presented in Tables 2 and 3, respectively, which were similar in all the Pre-Ki-67 and Post-Ki-67 subgroups. When adjusting for clinicopathological prognostic factors, a high-Ki-67 level 


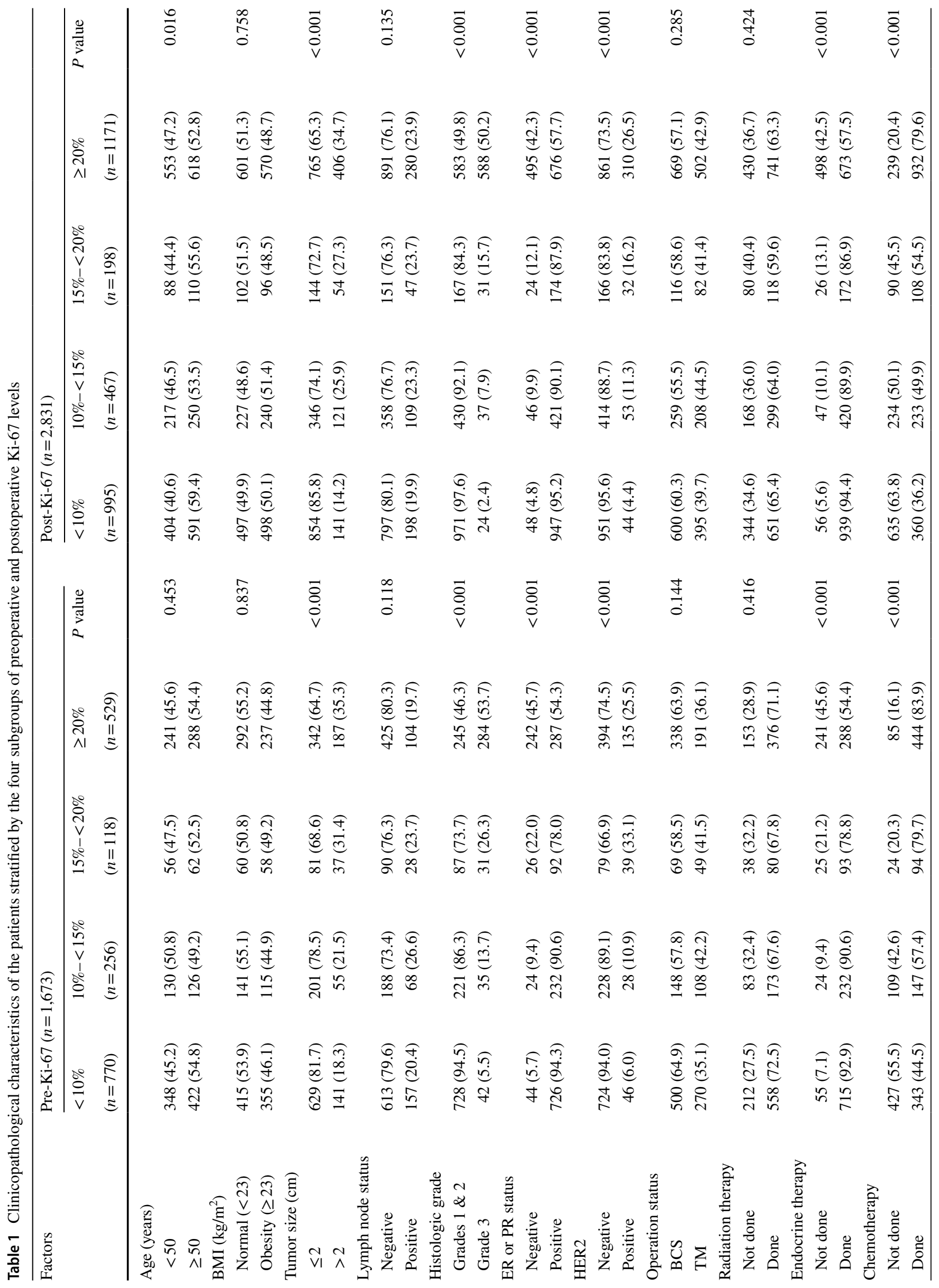


was determined to be an independent prognostic factor for both DFS and OS. However, the degree of hazard ratio was not proportional to Ki-67 levels. Larger tumor size and high nodal stage were also independent and significant prognostic factors for survival. Hormone receptor-positivity demonstrated an increased hazard ratio for DFS and OS without statistical significance. All systemic therapies had decreased hazard ratios for DFS and OS without statistical significance. Age was seen as an important prognostic factor for OS as compared with that for DFS.

Although only 327 (7.9\%) patients in the entire study population were examined for Ki-67 levels using both preoperative and postoperative specimens, we exploratively analyzed survival outcomes according to combined Pre-Ki-67 and Post-Ki-67 status. Given that patients with medium-low and medium-high levels of Ki-67 showed the worst outcomes, a cutoff point of Ki-67 was considered: $<15 \%$ (low) versus $\geq 15 \%$ (high). Of 327 cases, $43.7 \%$ and $32.4 \%$ had low and high-Ki-67 levels, respectively. However, $14.1 \%$ and $9.8 \%$ of patients had postoperatively increased and decreased Ki-67 levels, respectively. Figure 2 reveals the DFS and OS according to combined Ki-67 status; Pre-Ki-67 status may predict patient outcome even when discordant Ki-67 status existed in $23.9 \%$ of the cases.

\section{Discussion}

The motivation behind this study was to investigate whether the timing of measuring Ki-67 levels affected the accuracy of evaluating tumor progression and aggression, along with predicting patient prognosis. In several previous studies, it has been argued that the Ki-67 levels ascertained from biopsies and surgical specimens are related. However, most of the papers compared and analyzed biopsies and surgical specimens of the same patient and demonstrated that both Ki-67 levels were concordant [16, 17, 22]. In contrast, there have been studies that suggested that changes between CNB and surgical specimen affect the prognosis of patients. However, our study did not include patients who had undergone neoadjuvant chemotherapy because the prognosis was confirmed through an independent group of changes in the overall Ki-67 level and not just a change in one patient's Ki-67 level. Therefore, in this study, the Pre-Ki-67 (biopsy) and Post-Ki-67 (specimen) groups were independent without any overlap. We expected a proportional increase in the hazard ratio according to increased Ki-67 levels; however, since we confirmed that $\mathrm{Ki}-67$ is heterogeneous, there was no proportionate increase in the hazard ratio in the Pre-Ki-67 or Post-Ki-67 groups. However statistically, the hazard ratio increased more significantly in the Post-Ki-67 group than in the Pre-Ki-67 group; thus, it can be inferred that Ki-67 from specimens is superior for prognostic evaluation. Despite 

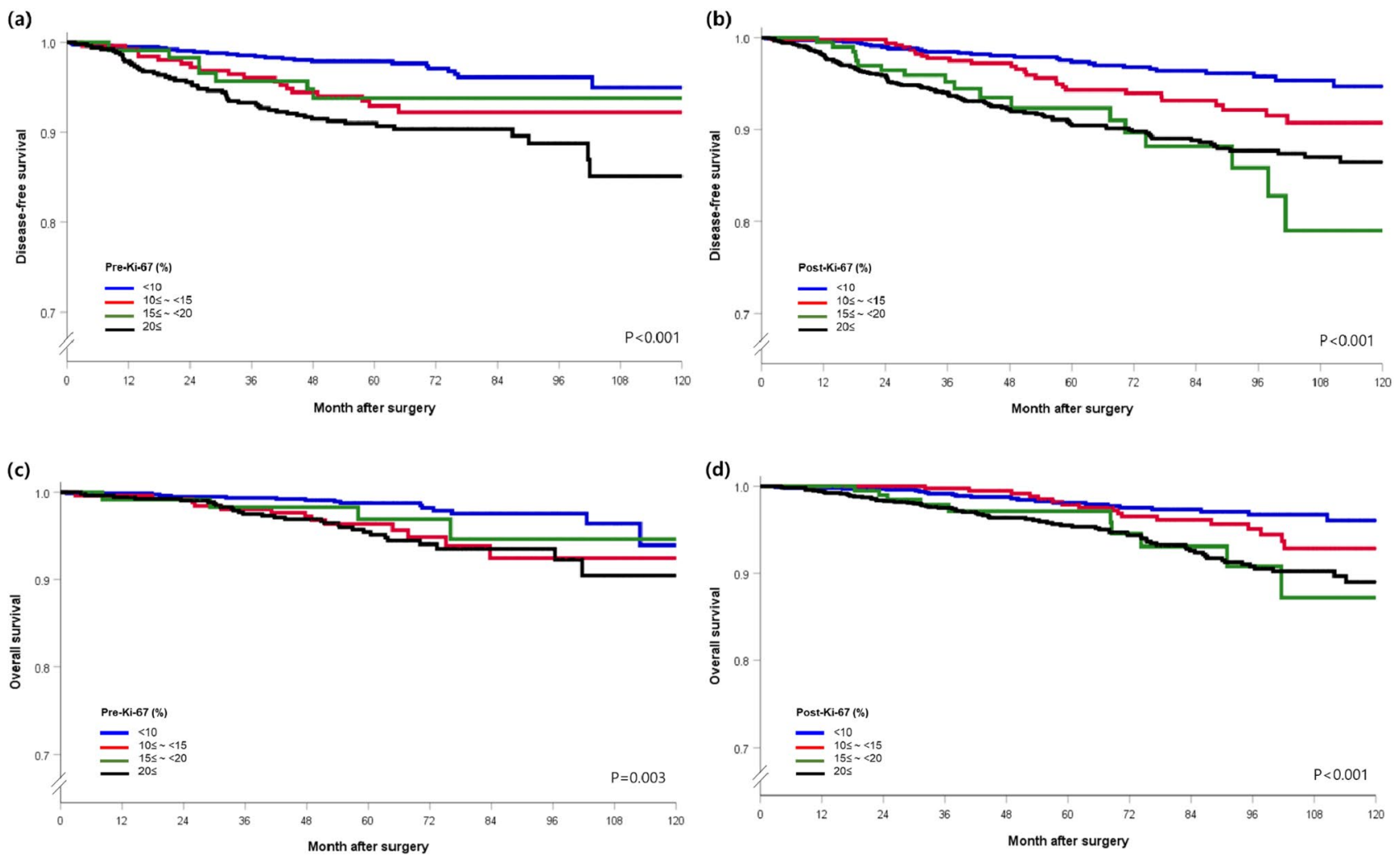

Fig. 1 Survival curves according to the four subgroups of Pre-Ki-67 and Post-Ki-67 levels (a) Disease-free survival in the Pre-Ki-67 group. (b) Disease-free survival in the Post-Ki-67 group. (c) Over-

these problems inevitably raised by the current design and nature of the study, independent groups showed similar prognostic patterns of Ki-67 levels, and it was proved that the Ki-67 level could predict the prognosis of breast cancer, regardless of the time of measurement. Moreover, this study showed that the Ki-67 index was related to different variables, such as larger tumor size, greater histologic grade, negative ER/PR status, and positive HER2 status [11, 12, $23,24]$. Therefore, the combination of Ki-67 and other variables should be further studied to validate our findings and develop a model that can predict prognosis more accurately.

Unlike previous prognostic studies, variables for therapeutic interventions were identified in the present study. In particular, with regard to local therapy along with proliferation, various other factors were considered. However, in the case of systemic therapy, the Ki-67 index tends to increase if a patient does not receive endocrine therapy and instead receives chemotherapy or biologic therapy. These results suggest that, regardless of the time of examination, the Ki-67 value indicates the final proliferative state of breast cancer and eventually indicates patient prognosis. Therefore, the treatment method can be modified depending on this result. A high-Ki-67 level reflects an advanced pathological state

all survival in the Pre-Ki-67 group. (d) Overall survival in the PostKi-67 group. Pre-Ki-67, preoperative Ki-67; Post-Ki-67, postoperative $\mathrm{Ki}-67$

and shows that the subtype is more likely to be a basal-like or HER2-only type than a luminal type [10, 22, 25, 26].

The results of this study demonstrated that the higher the Ki-67 values, the higher the probability of receiving more aggressive treatment. After it was established that Ki-67 levels play a prognostic role in breast cancer, Cheang et al. proposed a cutoff value for $\mathrm{Ki}-67$ as a prognostic factor and they evaluated this cutoff value for Ki-67 in several studies $[7,11,12,27,28]$. However, the methods of inspection and evaluation were insufficient due to variability between institutions, which precluded this cutoff from being included in a verified clinical guideline [9]. Recently, as inspection methods and evaluations have become automated, the objectivity of Ki-67 values has been secured to some extent; however, it is challenging to ensure standardization across all institutions $[29,30]$. Our study proves that Ki-67 level is valuable as a prognostic factor despite such issues.

Many related papers have already evaluated the cutoff value of Ki-67 and this has also been discussed at the St. Gallen conference $[11,12,15]$. The reason why the sub-classification of the Ki-67 index in our analysis was conducted around $10 \%, 15 \%$, and $20 \%$ is that in many studies related to $\mathrm{Ki}-67$ cutoff values, the point for the cutoff is reported to 
Table 2 Multivariate analysis for DFS according to Pre-Ki-67 and Post-Ki-67

\begin{tabular}{|c|c|c|c|c|c|c|}
\hline \multirow[t]{2}{*}{ Factor } & \multicolumn{3}{|l|}{ Pre-Ki-67 } & \multicolumn{3}{|l|}{ Post-Ki-67 } \\
\hline & Hazard ratio & $95 \% \mathrm{CI}$ & $P$ value & Hazard ratio & $95 \% \mathrm{CI}$ & $P$ value \\
\hline \multicolumn{7}{|l|}{ Ki-67 (\%) } \\
\hline$<10$ & 1 & & & 1 & & \\
\hline $10-<15$ & 2.227 & $1.194-4.154$ & 0.012 & 1.675 & $1.002-2.802$ & 0.049 \\
\hline $15-<20$ & 1.830 & $0.771-4.342$ & 0.171 & 3.009 & $1.666-5.433$ & $<0.001$ \\
\hline$\geq 20$ & 2.876 & $1.628-5.081$ & $<0.001$ & 2.340 & $1.452-3.693$ & $<0.001$ \\
\hline \multicolumn{7}{|l|}{ Age (years) } \\
\hline$<50$ vs. $\geq 50$ & 1.053 & $0.702-1.579$ & 0.803 & 1.234 & $0.918-1.658$ & 0.163 \\
\hline \multicolumn{7}{|l|}{ Tumor size $(\mathrm{cm})$} \\
\hline$\leq 2$ vs. $>2$ & 2.398 & $1.569-3.665$ & $<0.001$ & 1.586 & $1.164-2.162$ & 0.003 \\
\hline \multicolumn{7}{|l|}{ Lymph node status } \\
\hline Negative vs. Positive & 1.791 & $1.106-2.901$ & 0.018 & 1.817 & $1.284-2.570$ & 0.001 \\
\hline \multicolumn{7}{|l|}{ Histologic grade } \\
\hline Grades 1 and 2 vs. 3 & 0.904 & $0.549-1.488$ & 0.690 & 1.429 & $0.983-2.078$ & 0.059 \\
\hline \multicolumn{7}{|l|}{ ER/PR status } \\
\hline Negative vs. Positive & 1.217 & $0.261-5.669$ & 0.803 & 1.109 & $0.361-3.407$ & 0.952 \\
\hline \multicolumn{7}{|l|}{ HER2 status } \\
\hline Negative vs. Positive & 0.755 & $0.439-1.298$ & 0.310 & 0.633 & $0.421-0.952$ & 0.028 \\
\hline \multicolumn{7}{|l|}{ Operation status } \\
\hline BCS vs. TM & 1.147 & $0.595-2.211$ & 0.682 & 1.443 & $0.925-2.249$ & 0.106 \\
\hline \multicolumn{7}{|l|}{ Radiation therapy } \\
\hline Not done vs. Done & 0.531 & $0.280-1.005$ & 0.052 & 0.646 & $0.421-0.990$ & 0.045 \\
\hline \multicolumn{7}{|l|}{ Endocrine therapy } \\
\hline Not done vs. Done & 0.556 & $0.122-2.538$ & 0.449 & 0.579 & $0.192-1.749$ & 0.333 \\
\hline \multicolumn{7}{|l|}{ Chemotherapy } \\
\hline Not done vs. Done & 0.958 & $0.561-1.638$ & 0.877 & 0.893 & $0.608-1.313$ & 0.566 \\
\hline
\end{tabular}

$C I$ confidence interval, $E R$ estrogen receptor, $P R$ progesterone receptor, HER2 human epidermal growth factor receptor type 2, BCS breast-conserving surgery, $T M$ total mastectomy, $D F S$ disease-free survival be around $10-20 \%$ and we believed that significant changes in $\mathrm{Ki}-67$ wound occur within that range $[11,12,27,28$, 31]. In this study, a hazard ratio was not proportionate to the Ki-67 level. This is another reason why it is difficult to determine the cutoff value of Ki-67. Nevertheless, different prognostic management studies must be conducted considering a Ki-67 cutoff value of each institution in breast cancer patients under the same conditions in clinical practice to generate robust results. However, our main purpose is not to evaluate the prognosis through such a cutoff, but to show that, as an independent group, Ki-67 examined by Biopsy and surgical specimen has similar trends.

Our study had certain limitations related to the interpretation of results. The medium-low and medium-high groups had smaller sample sizes than that of the other Ki-67 subgroups; therefore, trend interpretation may be limited. Additionally, variables with similar properties to the Ki-67 level, particularly histologic grade, differed from the general results due to their overlapping with each other. As with other studies, a similar trend was observed in patients who underwent both tests and were similar to those in previous studies with a different prognosis according to the results of Ki-67 from biopsies rather than specimens. However, a limitation of this study is that accurate analysis is difficult due to the small sample size. Another inherent limitation was its single-center, retrospective study design.

Although many IHC biomarkers such as Ki-67 have been compared in biopsy and surgical specimens, multigene assays are also compared. Although it is not yet a study with the same concept as our study, there are studies that compare the results of the Oncotype DX from biopsy with from the surgical specimen and suggest that Oncotype DX from biopsy results is also concordant and reliable with the surgical specimen [32, 33]. As our future study, based on this study, we will begin prognostic study in luminal type and comparison of prognosis between Ki-67 and Oncotype DX or other multigene analysis.

However, to the best of our knowledge, this is one of the largest retrospective studies on the prognostic effectiveness of $\mathrm{Ki}-67$; in addition to being a retrospective, non-metastatic breast cancer population without any prior selection bias. Additionally, performing pathological and biomarker 
Table 3 Multivariate analysis for OS according to Pre-Ki-67 and Post-Ki-67

\begin{tabular}{|c|c|c|c|c|c|c|}
\hline \multirow[t]{2}{*}{ Factor } & \multicolumn{3}{|l|}{ Pre-Ki-67 } & \multicolumn{3}{|l|}{ Post-Ki-67 } \\
\hline & Hazard ratio & $95 \% \mathrm{CI}$ & $P$ value & Hazard ratio & $95 \% \mathrm{CI}$ & $P$ value \\
\hline \multicolumn{7}{|l|}{ Ki-67 (\%) } \\
\hline$<10$ & 1 & & & 1 & & \\
\hline $10-<15$ & 2.640 & $1.242-5.611$ & 0.012 & 1.412 & $0.747-2.670$ & 0.288 \\
\hline $15-<20$ & 1.668 & $0.539-5.164$ & 0.375 & 2.382 & $1.113-5.095$ & 0.025 \\
\hline$\geq 20$ & 2.310 & $1.117-4.781$ & 0.024 & 2.139 & $1.236-3.701$ & 0.007 \\
\hline \multicolumn{7}{|l|}{ Age (years) } \\
\hline$<50$ vs. $\geq 50$ & 1.739 & $1.019-2.967$ & 0.042 & 1.412 & $0.974-2.048$ & 0.068 \\
\hline \multicolumn{7}{|l|}{ Tumor size $(\mathrm{cm})$} \\
\hline$\leq 2$ vs. $>2$ & 3.107 & $1.782-5.419$ & $<0.001$ & 1.749 & $1.187-2.577$ & 0.005 \\
\hline \multicolumn{7}{|l|}{ Lymph node status } \\
\hline Negative vs. Positive & 2.362 & $1.281-4.356$ & 0.006 & 2.486 & $1.624-3.806$ & $<0.001$ \\
\hline \multicolumn{7}{|l|}{ Histologic grade } \\
\hline Grades 1 and 2 vs. 3 & 0.892 & $0.472-1.683$ & 0.724 & 1.569 & $0.990-2.488$ & 0.055 \\
\hline \multicolumn{7}{|l|}{ ER/PR status } \\
\hline Negative vs. Positive & 1.474 & $0.242-8.967$ & 0.674 & 1.280 & $0.358-4.576$ & 0.704 \\
\hline \multicolumn{7}{|l|}{ HER2 status } \\
\hline Negative vs. Positive & 0.722 & $0.355-1.469$ & 0.369 & 0.707 & $0.428-1.166$ & 0.174 \\
\hline \multicolumn{7}{|l|}{ Operation status } \\
\hline BCS vs. TM & 1.194 & $0.538-2.646$ & 0.663 & 1.381 & $0.789-2.416$ & 0.259 \\
\hline \multicolumn{7}{|l|}{ Radiation therapy } \\
\hline Not done vs. Done & 0.684 & $0.315-1.487$ & 0.338 & 0.576 & $0.338-0.983$ & 0.043 \\
\hline \multicolumn{7}{|l|}{ Endocrine therapy } \\
\hline Not done vs. Done & 0.379 & $0.064-2.236$ & 0.284 & 0.489 & $0.140-1.714$ & 0.264 \\
\hline \multicolumn{7}{|l|}{ Chemotherapy } \\
\hline Not done vs. Done & 0.731 & $0.370-1.447$ & 0.369 & 0.600 & $0.374-0.963$ & 0.034 \\
\hline
\end{tabular}

$C I$ confidence interval, $E R$ estrogen receptor, $P R$ progesterone receptor, HER2 human epidermal growth factor receptor type 2, BCS breast-conserving surgery, $T M$ total mastectomy, $O S$ overall survival (a)

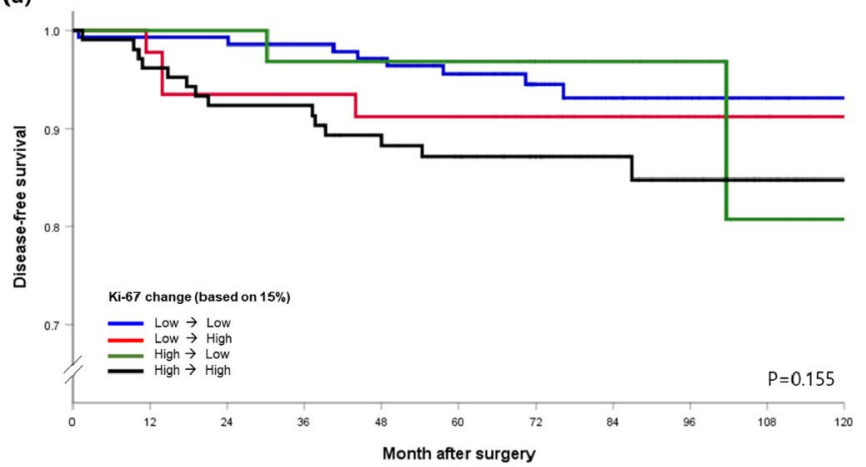

(b)

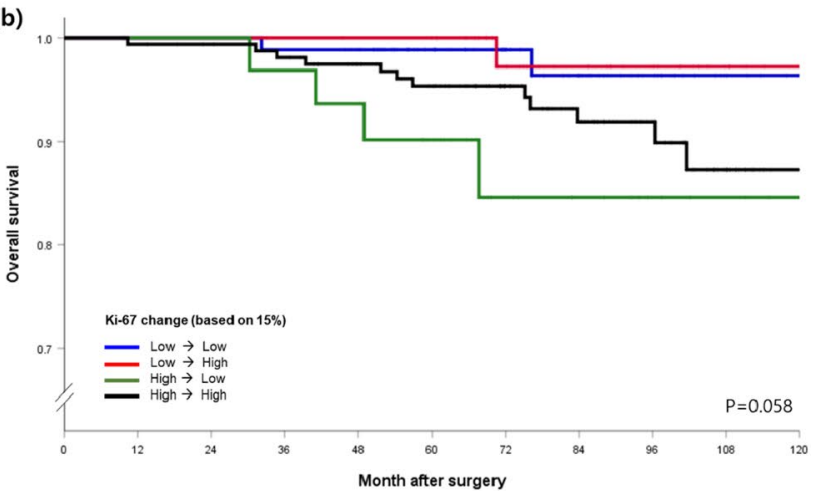

Fig. 2 Survival curves according to Ki-67 level change. (a) Disease-free survival. (b) Overall survival

evaluation in a single, well trained, and accredited laboratory was another advantage of this study, which demonstrated realistic values of IHC in clinical practice [16, 24]. Moreover, the fact that we considered variables related to treatment also differentiates the present study from previous ones.

\section{Conclusion}

We found that the Ki-67 level is an important independent prognostic factor that is more accurate than any other 
factor, regardless of the timing of Ki-67 testing, i.e., tested preoperatively or postoperatively. In addition, even if there are methodological and analytical problems related to Ki-67 testing, evidently, there are no problems in clinical practice. And it is difficult to know which timing of $\mathrm{Ki}-67$ testing is superior. However, considering the independent comparisons of groups that had only one test and groups that had both tests, it seems to be advantageous for breast cancer treatment to check the Ki-67 level preoperatively. In recent years, breast cancer treatment has become diverse, and early diagnosis and evaluation of disease are highly beneficial for recovery. Therefore, we recommend measuring the Ki-67 level in the preoperative period and measuring Pre-Ki-67 levels, which are currently measured with CNB and VAB specimens, as good clinical practice. However, it should be considered that the Ki-67 level observed in specimens may be slightly more accurate in reflecting the prognosis.

\section{Acknowledgements None.}

Author's contributions SBC was responsible for conceptualization, methodology, software, formal analysis, investigation, writing the original manuscript, reviewing and editing, and visualization; JMP, JHA, JG, JK, HSP, SIK, and BP were responsible for data curation; SP was responsible for conceptualization, resources, data curation, reviewing and editing the manuscript, supervision, and project administration. All authors read and approved the final manuscript.

Funding No funding was received for conducting this study.

Data availability The datasets generated and/or analyzed during the current study are available from the corresponding author on reasonable request.

\section{Declarations}

Conflict of interest The authors declare that they have no conflict of interest.

Ethical approval This retrospective chart review study involving human participants was in accordance with the ethical standards of the institutional and national research committee and with the 1964 Helsinki Declaration and its later amendments or comparable ethical standards. The Institutional Review Board of Severance Hospital, Seoul, Republic of Korea (IRB No. 4-2020-1293) approved this study. The requirement for informed consent was waived due to the retrospective nature of the study.

Consent to participate The requirement for informed consent was waived due to the retrospective nature of the study.

Consent for publish Not applicable.

Open Access This article is licensed under a Creative Commons Attribution 4.0 International License, which permits use, sharing, adaptation, distribution and reproduction in any medium or format, as long as you give appropriate credit to the original author(s) and the source, provide a link to the Creative Commons licence, and indicate if changes were made. The images or other third party material in this article are included in the article's Creative Commons licence, unless indicated otherwise in a credit line to the material. If material is not included in the article's Creative Commons licence and your intended use is not permitted by statutory regulation or exceeds the permitted use, you will need to obtain permission directly from the copyright holder. To view a copy of this licence, visit http://creativecommons.org/licenses/by/4.0/.

\section{References}

1. Sørlie T et al (2001) Gene expression patterns of breast carcinomas distinguish tumor subclasses with clinical implications. Proc Natl Acad Sci 98(19):10869-10874

2. Sørlie T et al (2003) Repeated observation of breast tumor subtypes in independent gene expression data sets. Proc Natl Acad Sci 100(14):8418-8423

3. Paik S et al (2004) A multigene assay to predict recurrence of tamoxifen-treated, node-negative breast cancer. N Engl J Med 351(27):2817-2826

4. Goldhirsch A et al (2013) Personalizing the treatment of women with early breast cancer: highlights of the St Gallen International Expert Consensus on the Primary Therapy of Early Breast Cancer 2013. Ann Oncol 24(9):2206-2223

5. Arima $\mathrm{N}$ et al (2019) Ki-67 index value and progesterone receptor status can predict prognosis and suitable treatment in nodenegative breast cancer patients with estrogen receptor-positive and HER2-negative tumors. Oncol Lett 17(1):616-622

6. Goldhirsch A et al (2011) Strategies for subtypes-dealing with the diversity of breast cancer: highlights of the St Gallen International Expert Consensus on the Primary Therapy of Early Breast Cancer 2011. Ann Oncol 22(8):1736-1747

7. Urruticoechea A, Smith IE, Dowsett M (2005) Proliferation marker Ki-67 in early breast cancer. J Clin Oncol 23(28):7212-7220

8. Duchrow M et al (1996) Molecular characterization of the gene locus of the human cell proliferation-associated nuclear protein defined by monoclonal antibody Ki-67. Cell Prolif 29(1):1-12

9. Penault-Llorca F, Radosevic-Robin N (2017) Ki67 assessment in breast cancer: an update. Pathology 49(2):166-171

10. de Azambuja E et al (2007) Ki-67 as prognostic marker in early breast cancer: a meta-analysis of published studies involving 12,155 patients. Br J Cancer 96(10):1504-1513

11. Cheang MC et al (2009) Ki67 index, HER2 status, and prognosis of patients with luminal B breast cancer. J Natl Cancer Inst 101(10):736-750

12. Jung S-Y et al (2009) Ki-67 Expression Gives Additional Prognostic Information on St. Gallen 2007 and Adjuvant! Online Risk Categories in Early Breast Cancer. Ann Surg Oncol 16(5):1112-1121

13. Kontzoglou K et al (2013) Correlation between Ki67 and breast cancer prognosis. Oncology 84(4):219-225

14. Dowsett M et al (2011) Assessment of Ki67 in breast cancer: recommendations from the International Ki67 in Breast Cancer working group. J Natl Cancer Inst 103(22):1656-1664

15. Focke CM, van Diest PJ, Decker T (2016) St Gallen 2015 subtyping of luminal breast cancers: impact of different Ki67-based proliferation assessment methods. Breast Cancer Res Treat 159(2):257-263

16. Chu P-Y, et al (2016) Risk factors associated with discordant ki-67 levels between preoperative biopsy and postoperative surgical specimens in breast cancers. Plos One 11(3)

17. Ahn S et al (2018) Evaluation of Ki-67 index in core needle biopsies and matched breast cancer surgical specimens. Arch Pathol Lab Med 142(3):364-368

18. Al Nemer A (2017) The performance of Ki-67 Labeling index in different specimen categories of invasive ductal carcinoma of 
the breast using 2 scoring methods. Appl Immunohistochem Mol Morphol 25(2):86-90

19. Tendl-Schulz KA et al (2020) Factors influencing agreement of breast cancer luminal molecular subtype by Ki67 labeling index between core needle biopsy and surgical resection specimens. Virchows Arch 477(4):545-555

20. Hammond MEH et al (2010) American Society of Clinical Oncology/College of American Pathologists guideline recommendations for immunohistochemical testing of estrogen and progesterone receptors in breast cancer (unabridged version). Arch Pathol Lab Med 134(7):e48-e72

21. Wolff AC et al (2014) Recommendations for human epidermal growth factor receptor 2 testing in breast cancer: American Society of Clinical Oncology/College of American Pathologists clinical practice guideline update. Arch Pathol Lab Med 138(2):241-256

22. Gandini S et al (2014) Association of molecular subtypes with $\mathrm{Ki}-67$ changes in untreated breast cancer patients undergoing presurgical trials. Ann Oncol 25(3):618-623

23. Fasching PA et al (2019) Prognostic effect of Ki-67 in common clinical subgroups of patients with HER2-negative, hormone receptor-positive early breast cancer. Breast Cancer Res Treat 175(3):617-625

24. Kang Y-J et al (2019) Ki-67 Expression is a significant prognostic factor only when progesterone receptor expression is low in estrogen receptor-positive and HER2-negative early breast Cancer. J Oncol 2019:7386734

25. Zhu X, et al. (2020) The prognostic and predictive potential of $\mathrm{Ki}-67$ in triple-negative breast cancer. Sci Rep 10(1)

26. Wu Q et al (2019) Prognostic value of Ki-67 in patients with resected triple-negative breast cancer: a meta-analysis. Front Oncol 9:1068
27. Petrelli F et al (2015) Prognostic value of different cut-off levels of Ki-67 in breast cancer: a systematic review and meta-analysis of 64,196 patients. Breast Cancer Res Treat 153(3):477-491

28. Bustreo $\mathrm{S}$ et al (2016) Optimal Ki67 cut-off for luminal breast cancer prognostic evaluation: a large case series study with a longterm follow-up. Breast Cancer Res Treat 157(2):363-371

29. Saha $\mathrm{M}$ et al (2017) An advanced deep learning approach for $\mathrm{Ki}-67$ stained hotspot detection and proliferation rate scoring for prognostic evaluation of breast cancer. Sci Rep 7(1):1-14

30. Razavi S, et al (2018) An automated and accurate methodology to assess ki-67 labeling index of immunohistochemical staining images of breast cancer tissues. In: 2018 25th International Conference on Systems, Signals and Image Processing (IWSSIP). IEEE

31. Tashima R et al (2015) Evaluation of an Optimal Cut-Off Point for the Ki-67 Index as a Prognostic Factor in Primary Breast Cancer: A Retrospective Study. PLoS ONE 10(7):e0119565-e0119565

32. Orozco JIJ et al (2021) Is the 21-Gene recurrence score on core needle biopsy equivalent to surgical specimen in early-stage breast cancer? A comparison of gene expression between paired core needle biopsy and surgical specimens. Ann Surg Oncol 28(10):5588-5596

33. Qi P et al (2021) Concordance of the 21-gene assay between core needle biopsy and resection specimens in early breast cancer patients. Breast Cancer Res Treat 186(2):327-342

Publisher's Note Springer Nature remains neutral with regard to jurisdictional claims in published maps and institutional affiliations. 